\title{
Recent results from the LHCf and RHICf experiments
}

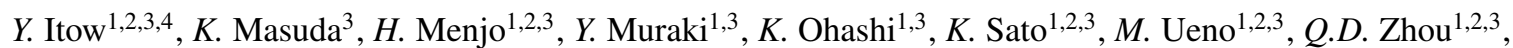
T. Sako ${ }^{1,2,5, *}, K$. Kasahara ${ }^{1,2,6}, T$. Suzuki ${ }^{1,2,6}, S$. Torii ${ }^{1,2,6}, T$. Tamura ${ }^{1,7}, N$. Sakurai ${ }^{1,2,8}, M$. Haguenauer ${ }^{1,9}$, W.C. Turner ${ }^{1,10}, O$. Adriani ${ }^{1,2,11,12}, E$. Berti ${ }^{1,2,11,12}$, L. Bonechi ${ }^{1,2,11}, M$. Bongi ${ }^{1,11,12}, R$. D'Alessandro ${ }^{1,2,11,12}, P$. Papini $^{1,11}$,

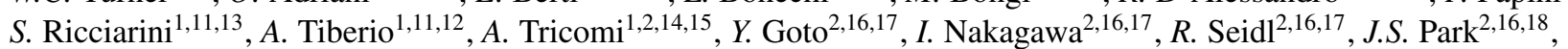

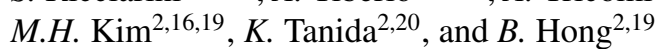

${ }^{1}$ The LHCf Collaboration

${ }^{2}$ The RHICf Collaboration

${ }^{3}$ Institute for Space-Earth Environment Research, Nagoya University, Nagoya, Japan

${ }^{4}$ Kobayashi-Maskawa Institute for the Origin of Particles and the Universe, Nagoya University, Nagoya, Japan

${ }^{5}$ Institute for Cosmic Ray Research, University of Tokyo, Kashiwa, Chiba, Japan

${ }^{6}$ Research Institute for Science and Engineering, Waseda University, Shinjuku, Tokyo, Japan

${ }^{7}$ Kanagawa University, Kanagawa, Japan

${ }^{8}$ Tokushima University, Tokushima, Japan

${ }^{9}$ Ecole-Polytechnique, Palaiseau, France

${ }^{10}$ LBNL, Berkeley, CA, USA

${ }^{11}$ INFN Section of Florence, Florence, Italy

${ }^{12}$ University of Florence, Florence, Italy

${ }^{13}$ IFAC-CNR, Florence, Italy

${ }^{14}$ INFN Section of Catania, Catania, Italy

${ }^{15}$ University of Catania, Catania, Italy

${ }^{16}$ Riken Nishina Center for Accelerator-Based Science, Saitama, Japan

${ }^{17}$ Riken BNL Research Center, Brookhaven National Laboratory, New York, USA

${ }^{18}$ Seoul National University, Seoul, South Korea

${ }^{19}$ Korea University, Seoul, South Korea

${ }^{20}$ Japan Atomic Energy Agency, Ibraki, Japan

\begin{abstract}
The Large Hadron Collider forward and the Relativistic Heavy Ion Collider forward experiments measured forward particles produced in high-energy hadron collisions at the LHC and RHIC. Using compact calorimeters neutral particles produced in pseudorapidities $\eta>8.4$ and $\eta>6.0$ are observed by the respective experiments. Because the collision energies ranging from $0.51 \mathrm{TeV}$ to $13 \mathrm{TeV}$ correspond to the cosmic-ray equivalent energies of $10^{14}$ to $10^{17} \mathrm{eV}$, the measurements are important to understand the hadronic interaction relevant to extensive air shower measurements. This paper reviews recent results of LHCf and initial performance of RHICf that took data in the 2017 RHIC operation.
\end{abstract}

\section{Introduction}

Hadronic interaction is a key to understand the development of extensive air showers initiated by high-energy cosmic rays. Forward particle production is especially important because they carry a large fraction of the collision energy. However, because of their non-perturbative nature, a theoretical approach from the first principle is difficult [1]. Various phenomenological models are proposed but their uncertainty is a still one of the major sources of the air shower interpretations [2]. Experimental information of forward particle production using accelerators at as high an energy as possible and as wide an energy range as possible is essential.

\footnotetext{
*e-mail: sako@icrr.u-tokyo.ac.jp
}

The Large Hadron Collider forward (LHCf) and the Relativistic Heavy Ion Collider forward (RHICf) experiments are dedicated to measure neutral particles emitted around zero degree of hadron collisions at the LHC and RHIC, respectively. Their major objectives are to determine the differential production cross sections of photons, neutrons and neutral pions. Their unique phase space coverage provides new information about the high-energy hadronic interactions.

\section{The LHCf and RHICf experiments}

A schematic view of the LHCf/RHICf installation locations with respect to the other structures around the interaction points is shown in Fig.1. Detail of each experiment is explained in the following sections. 


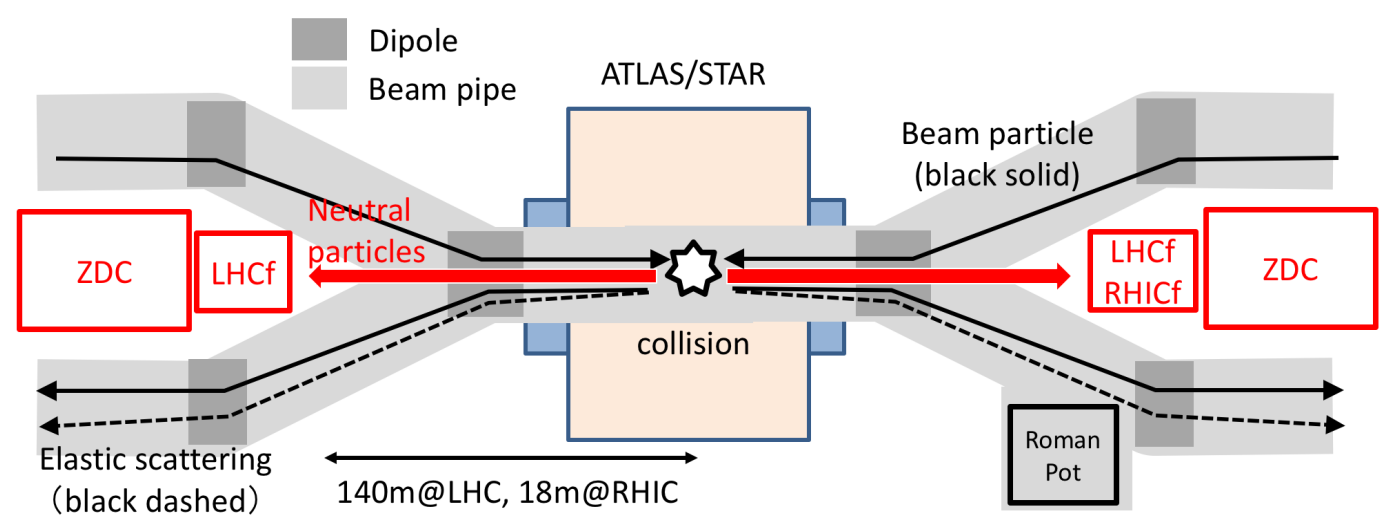

Figure 1. Schematic view of the LHCf and RHICf installation locations. Two LHCf detectors were installed either side of the interaction point (IP) and they shared the IP with ATLAS. RHICf covered only one side sharing the IP with STAR. RHICf operated with ZDCs at either side and also with roman pot detectors.
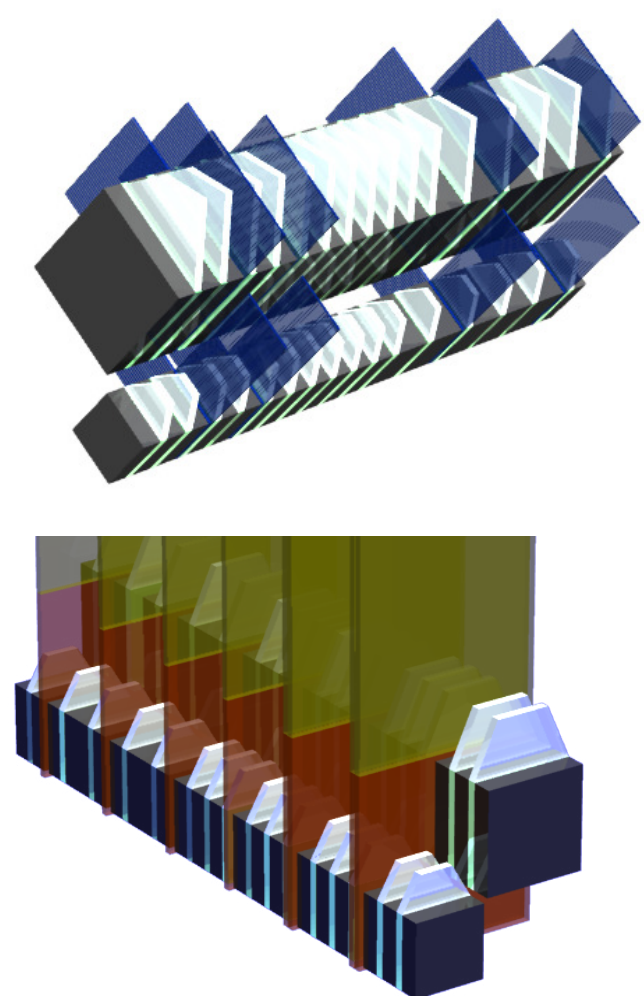

Figure 2. Schematic views of the LHCf Arm1 (top) Arm2 (bottom) detectors containing two sampling calorimeters. Grey part represents the Tungsten plates. Blue and brown parts represent the scintillating fiber bundles and silicon strip sensors, respectively. Sampling scintillators are interleaved with the Tungsten plates.

\subsection{LHCf}

LHCf installed two detectors called Arm1 and Arm2 at either side of the LHC interaction point 1 (IP1) where the ATLAS experiment is located. At $140 \mathrm{~m}$ from the IP, a single wide beam pipe facing to the IP is separated to smaller two pipes. By installing compact detectors in the
$10 \mathrm{~cm}$ gap between these two pipes, neutral particles emitted around zero degrees can be observed. Charged particles are swept away by dipole magnet between the IP and the detector location. Each of Arm1 and Arm2 contains two compact sampling calorimeters. The transverse dimensions of the calorimeters in Arm 1 are $20 \mathrm{~mm} \times 20 \mathrm{~mm}$ and $40 \mathrm{~mm} \times 40 \mathrm{~mm}$ while they are $25 \mathrm{~mm} \times 25 \mathrm{~mm}$ and $32 \mathrm{~mm} \times 32 \mathrm{~mm}$ in Arm2. Schematic views of the detectors are shown in Fig.2. Each calorimeter is composed of 16 sampling scintillators and Tungsten plates of total 44 radiation lengths thickness. Position sensors are inserted to measure the lateral shape of the showers developed in the calorimeters. Scintillating fiber bundle and silicon strip sensors are used in Arm1 and Arm2, respectively. More details of the detector and their calibration are found in $[3,4]$. It is noted that the sampling scintillators and scintillating fibers were replaced with GSO scintillators and GSO bars, respectively, from the operation of 2015 for radiation hardness $[5,6]$.

LHCf realized its data taking since the first collision of the LHC in 2009 [7]. Since then, collision data were taken at different collision energies and different colliding particles. Most operations were carried out with dedicated collision conditions, i.e., under low luminosity to avoid pileup and high $\beta^{*}$ to reduce the angular spread due to the beam divergence. Since 2015, the trigger signals of LHCf were sent to ATLAS and ATLAS recorded the events accordingly. This enables us to analyze low mass diffraction events as discussed in Sec.3.2. A summary of the LHCf operations and publications are given in Table1.

\subsection{RHICf}

RHICf was proposed to extend LHCf to the lower energy collisions realized at RHIC [8]. RHIC interaction points can also host so-called zero degree calorimeters in the $10 \mathrm{~cm}$ gap of the two beam pipes behind the dipole magnet. The main advantage with respect to the LHC is the distance from the interaction point being $18 \mathrm{~m}$ instead of $140 \mathrm{~m}$ at the LHC. Because the maximum acceptance in 
Table 1. Summary of the LHCf operations and publications. Year 2017 is the operation of RHICf. Columns $\gamma, \mathrm{n}, \pi^{0}$ designate the analysis results of photons, neutrons and $\pi^{0}$ 's.

\begin{tabular}{ccccccc}
\hline Year & $\sqrt{s_{N N}}[\mathrm{TeV}]$ & Particles & Detector & \multicolumn{3}{c}{ References } \\
& & & & $\gamma$ & $\mathrm{n}$ & $\pi^{0}$ \\
\hline 2009,2010 & 0.9 & $\mathrm{p}-\mathrm{p}$ & Arm1 and Arm2 & {$[9]$} & & \\
2010 & 7 & $\mathrm{p}-\mathrm{p}$ & Arm1 and Arm2 & {$[10]$} & {$[11]$} & {$[12][14]$} \\
2013 & 2.76 & $\mathrm{p}-\mathrm{p}$ & Arm2 & & & {$[13][14]$} \\
2013 & 5.02 & $\mathrm{p}-\mathrm{Pb}$ & Arm2 & & & {$[13][14]$} \\
2015 & 13 & $\mathrm{p}-\mathrm{p}$ & Arm1 and Arm2 & {$[15][16]$} & {$[19]$} & \\
2016 & 5.02 and 8.16 & $\mathrm{p}-\mathrm{Pb}$ & Arm2 & & & \\
2017 & 0.51 (RHIC) & polarized p-p & Arm1 & & & \\
\hline
\end{tabular}

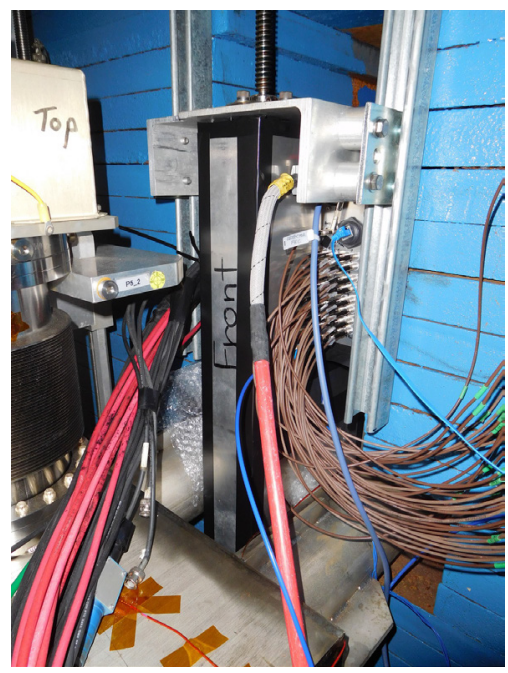

Figure 3. Photo of the RHICf detector, former LHCf Arm1, installed near the STAR interaction point. There is a structure allowing the detector to move vertically.

the transverse momentum $p_{T, \max }$ is limited by the geometrical angular limit $\theta_{\max }$ as

$$
p_{T, \max }=\frac{1}{2} \sqrt{s} \sin \theta_{\max },
$$

the close distance to IP (larger $\theta_{\max }$ ) can compensate with small $\sqrt{s}$. Then RHICf can cover a comparable $x_{F}-p_{T}$ phase space to LHCf. Here $x_{F}$ is Feynman $\mathrm{x}$ defined as $x_{F}=2 p_{z} / \sqrt{s}$.

The LHCf Arm1 detector was transferred to the BNL after the 2015 LHCf operation. With a small modification to fit the available space, the detector was installed $18 \mathrm{~m}$ west from the interaction point of the STAR experiment as shown in Fig.3 . Dedicated operation time was assigned at the end of RUN2017 and RHICf successfully collected collision data from 24 to 28 June. During this period, proton beams were colliding with $\sqrt{s}=510 \mathrm{GeV}$ and $\beta^{*}=8 \mathrm{~m}$. High $\beta^{*}$ reduced the luminosity down to $10^{31} \mathrm{~cm}^{-2} \mathrm{~s}^{-1}$ and the effect of pileup was reduced to a negligible level considering about $3 \%$ detector acceptance to inelastic collisions. Similar to the LHCf case, the RHICf trigger signals were sent to STAR and STAR recorded data accordingly. Initial performance of the RHICf detector obtained from these data are presented in Sec.4.
In the RHICf operation, spin directions of the proton beams were transversely polarized. Experiments at RHIC discovered asymmetrical production of forward particles with respect to the direction of the beam polarization. Using the better position resolution and the unique phase space coverage of the RHICf detector than the previous experiments, RHICf aims 1) to measure the $p_{T}$ dependence of the forward neutron asymmetry and 2) to discover or best constrain the forward $\pi^{0}$ asymmetry.

\section{Recent results of LHCf}

\subsection{Inclusive photons at $\sqrt{s}=13 \mathrm{TeV}$}

LHCf analyzed data taken in $13 \mathrm{TeV}$ p-p collisions and published differential cross sections of inclusive photon production [15]. The analysis covered pseudorapidity regions of $\eta>10.94$ and 8.99> $\eta>8.81$. The experimental results are compared with the cross sections predicted by major generators like QGSJET II-04, EPOSLHC, SIBYLL 2.3, DPMJET 3.06 and PYTHIA 8.212. The first three are usually used in the analyses of cosmicray air showers and are versions updated using the LHC Run1 results. The last one is a popular generator in the high-energy physics. EPOS-LHC shows the best agreement with the experimental data, but having a small excess in the highest energy region. PYTHIA also shows a general agreement up to the medium energy $(\sim 3 \mathrm{TeV})$, but exhibiting a larger excess than EPOS at higher energy. QGSJET shows a good agreement in the spectral shape in the high rapidity region but with a systematic deficit. The deficit increases in the lower rapidity region. SIBYLL also shows a deficit in the high rapidity region, but, in contrast to QGSJET, it has a significant excess in the low rapidity region. DPMJET always overpredicts the cross section in whole rapidity and energy regions.

Differences of predictions relative to the experimental data are similar to the results found in the $7 \mathrm{TeV}$ analysis [10], or are even exaggerated. Because of the twice higher collision energy, the same rapidity selected in these analyses corresponds to the twice larger transverse momentum. Wider phase space coverage in the $13 \mathrm{TeV}$ analysis enables a deeper inspection of the particle production.

\subsection{Joint analysis with ATLAS for $13 \mathrm{TeV}$ photons}

One of the important processes in forward particle production is diffractive dissociation. Diffractive process has 

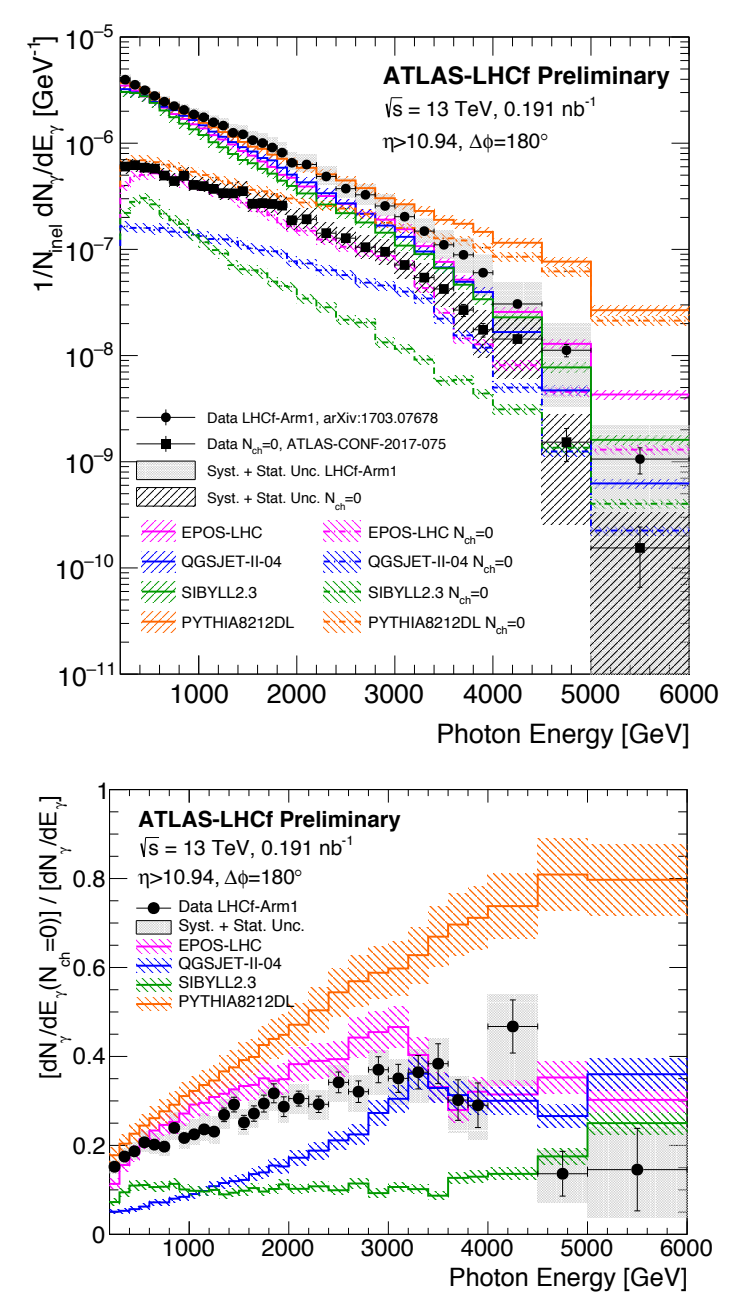

Figure 4. (Top) Energy spectra of inclusive and diffractive-like photons [16], (Bottom) Ratio of diffractive-like to inclusive photons.

a smaller multiplicity and produces more high-energy particles than non-diffractive processes. Experimentally because of the difficulty in the detection of especially low mass diffraction events, its cross section is not well understood. LHCf is a unique experiment sensitive to the low mass diffraction, however, it can not distinguish diffractive events from the other processes. The characteristic event shape of the diffractive events called rapidity gap is easily identified using the detectors covering the interaction point that is ATLAS at IP1. ATLAS and LHCf had common data taking in the $13 \mathrm{TeV}$ operation. In this operation, when LHCf observed high-energy particles in one of their detectors, ATLAS was forced to record their data even if they did not identify any particle in their volume.

Depending on the number of charged particles in the ATLAS tracker, LHCf classified their photon events into two categories [16] : diffractive-like events when no charged particles was identified in ATLAS, and nondiffractive-like events when any particles were identified in ATLAS. Using this criterion, LHCf can select diffractive events with $\log _{10}(\xi)<-5$, where $\xi=M^{2} / s$ with diffrac- tive mass $M$. A general discussion on the forward-central correlation was given in [17].

Fig.4 (top) shows photon cross sections in the $\eta>10.94$ range for inclusive (filled circles) and diffractivelike (filled squares) events. As expected, the diffractive events exhibit a harder spectrum than the inclusive events. Predictions from various generators are also shown for two event categories. It is found that PYTHIA (orange lines) overpredicts the diffractive photons while the QGSJET (blue) and SIBYLL (green) strongly suppress. The relative production rates of diffractive-like events to the inclusive events are shown in Fig.4 (bottom) together with the predictions. EPOS (magenta) shows best agreement with data both in the absolute value and the energy dependence.

\subsection{Inclusive neutrons at $\sqrt{s}=13 \mathrm{TeV}$}

Though the detection efficiency and the energy resolution of the LHCf calorimeters for hadronic showers are limited to $\sim 70 \%$ and $\sim 40 \%$ [18], respectively, cross sections for the forward neutron production were also analyzed by using only Arm2 data [19]. Differential cross sections in two rapidity regions, $\eta>10.76$ and $9.22>\eta>8.99$, after unfolding the energy resolution smearing are shown in Fig. 5 together with predictions by generators. A remarkable peak at $5 \mathrm{TeV}$, or $x_{F} \sim 0.8$, in $\eta>10.76$ was also reported by experiments at ISR [20], PHENIX at RHIC [21] and LHCf $7 \mathrm{TeV}$ analysis [11], for collision energies ranging from $30 \mathrm{GeV}$ to $7 \mathrm{TeV}$. This peak structure is not explained by any generators studied here. PHENIX reported a scaling of the $x_{F}$ distributions from $\sqrt{s}=30 \mathrm{GeV}$ to $200 \mathrm{GeV}$ when they compared in same $p_{T}$ range [21]. Similar study by LHCf is now on going.

The spectrum at lower rapidity also shows a peak structure but at a much lower energy. In contrast to the high rapidity result, the peak energy is well reproduced by most generators. Among them SIBYLL 2.3 best reproduces the absolute cross section. It was not the case when LHCf compared $7 \mathrm{TeV}$ data with SIBYLL 2.1 [11]. The improvement of SIBYLL is also found in the $\eta>10.76$ region.

A dramatic change in spectral shape between two rapidity regions is an interesting topic. Expansion of the analysis phase space is in progress using the Arm1 data and the data taken at the different detector positions.

\section{Initial performance of RHICf}

\subsection{Operation and Statistics}

Accumulated number of events during the 2017 RHICf operation is shown in Fig.6. A trigger was generated when any 3 successive sampling layers detect energy greater than $45 \mathrm{MeV}$. Using this shower trigger, 'L3T (Shower)' in Fig.6, the RHICf detector has $100 \%$ efficiency for photons $>20 \mathrm{GeV}$. Hadronic showers initiated by neutrons are also triggered but with a higher threshold energy. Because the data taking speed of RHICf was limited up to $1 \mathrm{kHz}, \mathrm{L} 3 \mathrm{~T}$ (Shower) trigger was prescaled by a factor 10 to 30 and special triggers were introduced to enhance rare events. 

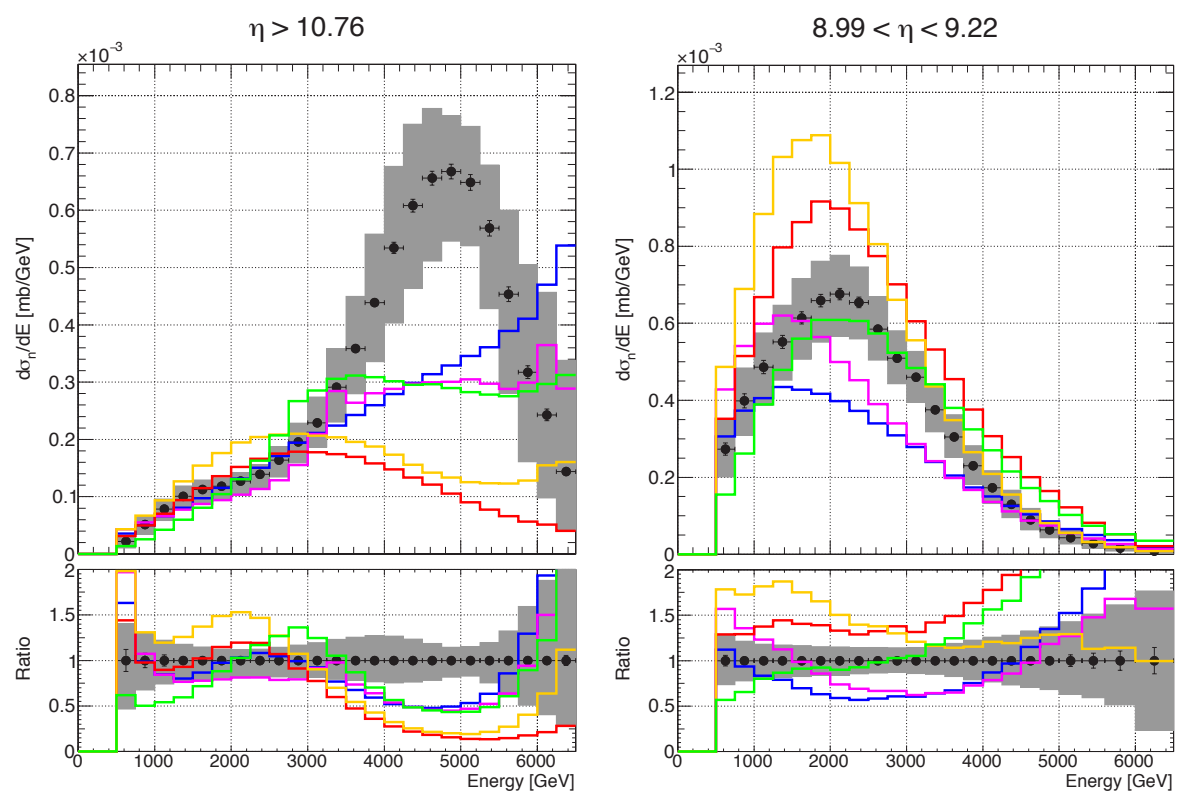

Figure 5. Differential neutron production cross sections at $\eta>10.76$ (left) and $9.22>\eta>8.99$ (right) in $13 \mathrm{TeV}$ p-p collisions measured by the LHCf Arm2 detector [19]. Colored histograms are predictions by QGSJET II-04 (blue), EPOS-LHC (magenta), DPMJET 3.06 (red), PYTHIA 8.212 (orange) and SIBYLL 2.3 (green) generators.

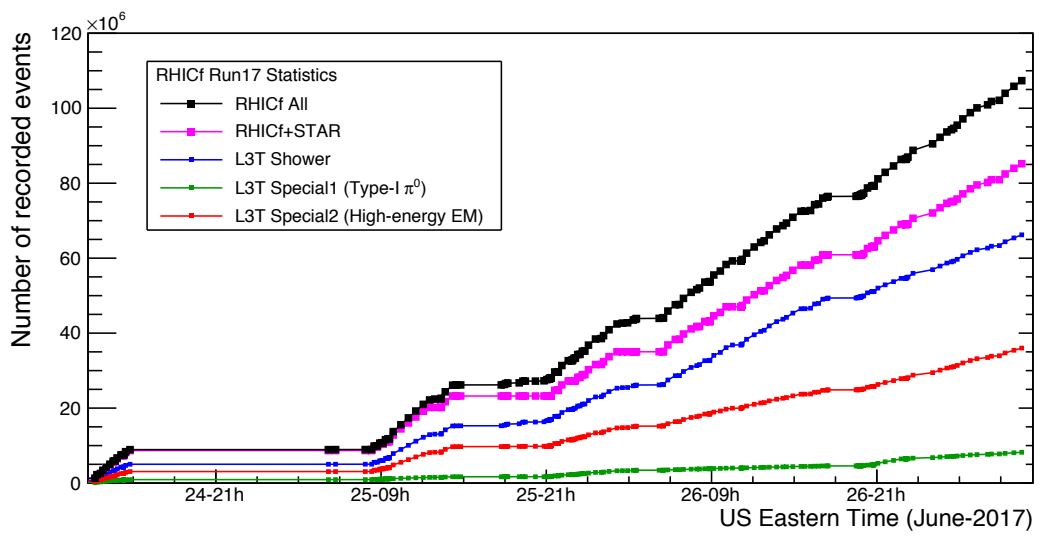

Figure 6. Accumulated number events during the RHICf operation in 2017. Definitions of various trigger mode are explained in the text.

'L3T Special1 (Type-I $\pi^{0}$ )' designates one of the special triggers that is issued when two calorimeters identify electromagnetic showers simultaneously. This trigger can enhance the $\pi^{0} \rightarrow \gamma \gamma$ events which allow us to reconstruct four momenta of $\pi^{0}$ produced and decayed at the interaction point. The other special trigger 'L3T Special2 (Highenergy EM)' is designed to selectively trigger high-energy electromagnetic showers. This is realized by setting the threshold of the $4^{\text {th }}$ sampling layer to be $500 \mathrm{MeV}$ instead of $45 \mathrm{MeV}$. This trigger is useful to not only enhance highenergy single photon events, but also high-energy $\pi^{0}$ because, at high energy, photons from $\pi^{0}$ decay enter in the same calorimeter that can not be detected by the 'L3T Special1 (Type-I $\pi^{0}$ )' trigger.
A total of $1.1 \times 10^{8}$ events were recorded by RHICf in 27.7 hours of data acquisition period. The RHICf trigger signal was sent to the STAR experiment and STAR also recorded data of their subdetectors such as TPC, TOF counters, Beam-Beam Counters, Zero Degree Calorimeter, and Roman Pot detectors. About $80 \%$ of RHICf triggered events were also recorded by STAR shown as 'RHICf+STAR' in Fig.6. This common data taking allows similar analysis performed between ATLAS and LHCf discussed in Sec.3.2, but new information are also available using ZDC and RPs.

Fig. 7 shows the correlation between energies observed in the RHICf detector and in the STAR ZDC detectors. The RHICf detector was installed in front of the west ZDC, then the shower particles leaked from the RHICf 

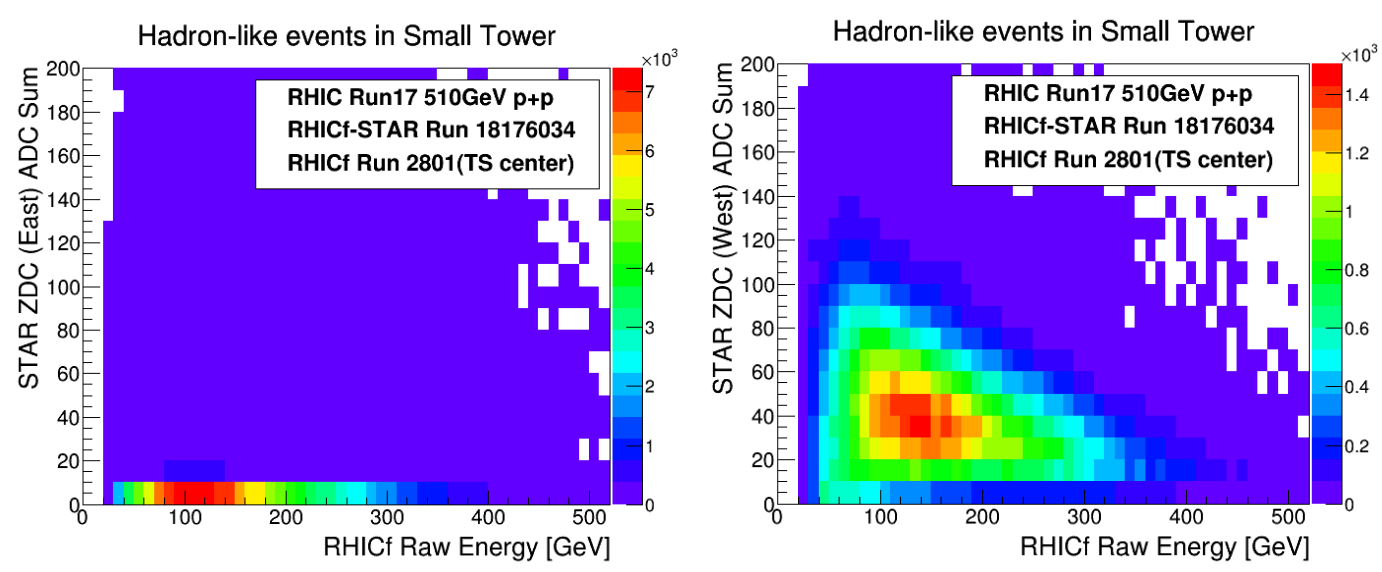

Figure 7. Correlation of observed energies in the RHICf detector and STAR ZDCs. Left (right) panel shows the result of the east (west) ZDC.

calorimeters can be collected by the ZDC having a larger acceptance and thickness. The left panel shows the result of ZDC at the east side of the STAR interaction point, while the right panel shows the west. In this analysis, deep penetrating showers, that is a characteristic feature of hadronic showers, in the RHICf detector were selected. Note that the RHICf energy exceeds the beam energy $255 \mathrm{GeV}$ because of the limited energy resolution for hadronic showers. Because the RHICf detector was installed in the west side of STAR, (anti)correlation is found only in the right panel (west). Anticorrelation is as expected because the ZDC compensates the leakage from RHICf. The result assures successful event matching in the RHICf-STAR joint operation.

\section{$4.2 \pi^{0}$ identification}

Identification of $\pi^{0}$ events is a key to confirm successful data taking. As discussed in Sec.4.1, $\pi^{0}$ 's are identified by reconstructing the invariant mass of photon pair events. Fig. 8 shows an invariant mass distribution obtained from the analysis of a $30 \mathrm{~min}$ long run. A clear peak near $140 \mathrm{MeV}$, that is an evidence of successful $\pi^{0}$ detection, is observed. A small shift from the real $\pi^{0}$ rest mass is an issue of a further energy calibration.

Fig.9 shows the number of $\pi^{0}$ candidates as a function of $x_{F}$ and $p_{T}$ after accumulating most of the available data. Because of the geometrical effect, operation at a single detector position can not cover the phase space shown in the figure. By moving the detector at three different vertical positions, coverage without gap was achieved. At the same time, a large number of events is detected even at the highest energy and $p_{T}$, where the production cross section rapidly drops. This is thanks to the 'L3T Special2 (High-energy EM)' trigger introduced in Sec.4.1.

\subsection{Polarized beam operation}

A characteristic feature of RHIC is to collide polarized beams. In terms of forward measurements, a strong asymmetrical production of neutrons was well known by vari-

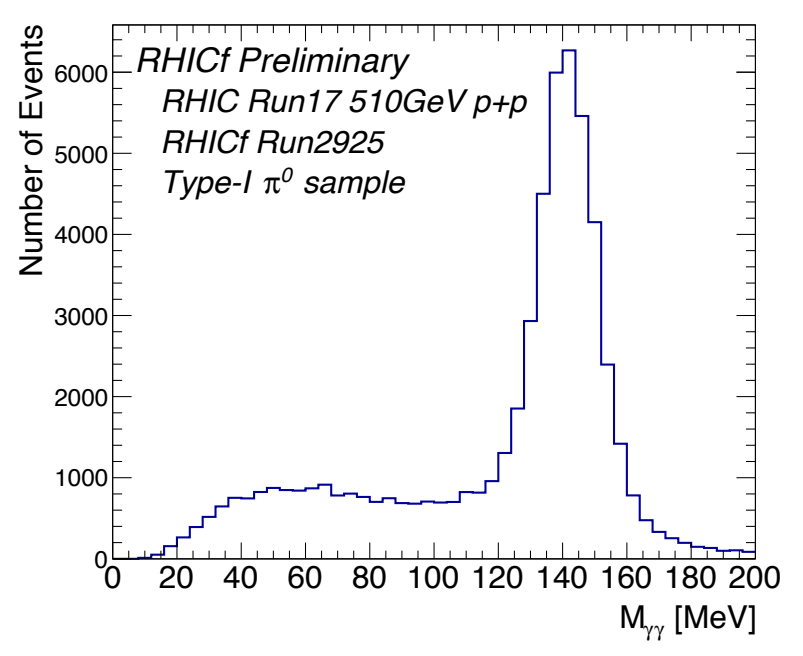

Figure 8. Invariant mass distribution of photon pair events.

ous RHIC experiments [21-23]. A theoretical explanation succeeds to explain the observed $p_{T}$ dependence of asymmetry by calculating the interference between fundamental pion exchange and $\mathrm{a}_{1}$ meson exchange [24]. However, because of the limited position resolution and comparison of data obtained at different collision energies, it is not obvious if the experimental results indicate really $p_{T}$ dependence. Thanks to the excellent position resolution of the RHICf detector, $\sim 1 \mathrm{~mm}$ instead of $10 \mathrm{~mm}$ in previous experiemnts, neutron asymmetry in the very forward region can be measured more precisely than the previous measurements. At the same time, wider $p_{T}$ with a single collision energy is covered by moving the detector position.

Forward $\pi^{0}$ asymmetry is also not well explored. STAR and PHENIX collaborations discovered up to $10 \%$ forward $\pi^{0}$ asymmetry [25] [26], but they covered only $p_{T}>1 \mathrm{GeV}$ that is in the regime of perturbative QCD. Only the upper limit of the very forward $\pi^{0}$ asymmetry, $p_{T}<1 \mathrm{GeV}$, is reported [27], but the sensitivity is not sufficient to discuss with $<1 \%$ accuracy. As shown in Fig.9, 




Figure 9. Number of $\pi^{0}$ candidate events as a function of $x_{F}$ and $p_{T}$.

RHICf recorded sufficient number of $\pi^{0}$ 's and is capable to determine asymmetry in different $x_{F}$ and $p_{T}$ bins. Preliminary results of $\pi^{0}$ asymmetry are presented in another paper at this symposium [28].

Beam polarization is usually monitored by ZDC and the Shower Maximum Detector (SMD) [21] through well known neutron asymmetry. Though RHICf obscured a part of the ZDC acceptance, the effect to the polarization measurement was negligible. Asymmetry amplitude and its direction measured by ZDC during the RHICf operation are shown in Fig.10. It is found that the amplitude and direction were stable. It must be noted that the polarization angle at $90^{\circ}$ is not a usual operation of RHIC. Because the RHICf detector can be moved only in the vertical direction, to maximize the asymmetry in this direction, beam polarization angle was turned $90^{\circ}$ from usual operation.

\section{Summary}

LHCf and RHICf are designed to study the forward particle productions in high-energy hadron collisions. The equivalent cosmic-ray energy covered by these experiments range from $10^{14} \mathrm{eV}$ to $10^{17} \mathrm{eV}$, that well overlaps with the energy range of air shower observations. LHCf has completed the initially proposed operation at the maximum LHC energy and continues data analyses. Inclusive cross sections of photons, neutrons and $\pi^{0}$ for various conditions are already published and are used to update the hadronic interaction models. Further analysis like joint analysis with ATLAS is ongoing. This analysis is expected to specify the source of difference between experimental data and models in terms of diffractive and non-diffractive processes.

RHICf covers the low energy side of air shower measurements. Data taking was completed successfully in 2017. Combining the data from LHCf and RHICf, the en-

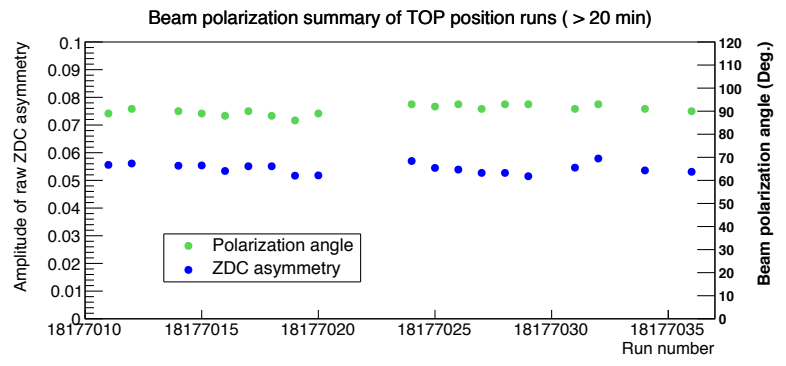

Figure 10. Amplitude and angle of the forward neutron asymmetry observed using ZDC during the RHICf operation.

ergy dependence of particle production can be studied and it is essential to extrapolate the knowledge to the $10^{20} \mathrm{eV}$ cosmic-ray interaction region. Asymmetrical particle production in the polarized beam collisions may give a hint to understand the fundamental process of hadronic interaction.

\section{Acknowledgements}

The LHCf members thank the CERN staff and ATLAS Collaboration for their essential contributions to the successful operation of LHCf. The RHICf members thank the BNL staff, STAR and PHENIX Collaborations for their essential contributions to the successful operation of RHICf. This work was partly supported by JSPS KAKENHI and the joint research program of the Institute for Cosmic Ray Research (ICRR), University of Tokyo. This work was also supported by Istituto Nazionale di Fisica Nucleare (INFN) in Italy. Parts of this work were performed using the computer resource provided by ICRR (University of Tokyo), CERN and CNAF (INFN). RHICf was partially supported by the U.S.-Japan Science and Technology Cooperation Program in High Energy Physics and by the National Research Foundation of Korea (No. 2016R1A2B2008505).

\section{References}

[1] K. Akiba et al., J. Phys. G: Nucl. Part. Phys. 43 110201 (2016).

[2] K.-H. Kampert and M. Unger, Astropart. Phys. 35 660-678 (2012).

[3] The LHCf Collaboration, JINST, 3 S08006 (2008).

[4] Y. Makino et al., JINST, 12 P03023 (2017).

[5] K. Kawade et al., JINST, 6 T09004 (2011).

[6] T. Suzuki et al., JINST, 8 T01007 (2013).

[7] The LHCf Collaboration, International. J. of Mod. Phys. A 281330036 (2013).

[8] The RHICf Collaboration, arXiv:1409.4860 [physics.ins-det].

[9] The LHCf Collaboration, Phys. Lett. B 715 298-303 (2012).

[10] The LHCf Collaboration, Phys. Lett. B 703 128-134 (2011). 
[11] The LHCf Collaboration, Phys. Lett. B 750 360-366 (2015).

[12] The LHCf Collaboration, Phys. Rev. D 86092001 (2012)

[13] The LHCf Collaboration, Phys. Rev. C 89065209 (2014).

[14] The LHCf Collaboration, Phys. Rev. D 94032007 (2016).

[15] The LHCf Collaboration, Phys. Lett. B 780, 233-239 (2018).

[16] The ATLAS and LHCf Collaborations, ATLASCONF-2017-075 (2017).

[17] Q.D. Zhou, Y. Itow, H. Menjo and T. Sako, Eur. Phys. J. C, 77:212 (2017).

[18] K.Kawade et al., JINST, 9, P03016 (2014).

[19] The LHCf Collaboration, CERN-EP-2018-239; arXiv:1808.09877v1 [hep-ex].
[20] J. Engler et al., Nucl. Phys. B84, 70 (1975).; W. Flauger and F. Monnig, Nucl. Phys. B 109, 347 (1976).

[21] The PHENIX Collaboration, Phys. Rev. D 88, 032006 (2013).

[22] Y. Fukao et al., Phys. Lett. B 650, 325-330 (2007). [hep-ex/0610030].

[23] The PHENIX Collaboration, J. Phys. Conf. Ser., 295, 012097 (2011).

[24] B. Z. Kopeliovich, I. K. Potashnikova, I. Schmidt and J. Soffer, Phys. Rev. D 84, 114012 (2011).

[25] The STAR Collaboration, Phys. Rev. Lett. 101, 222001 (2008).

[26] The PHENIX Collaboration, Phys. Rev. D 90, 012006 (2014).

[27] Y. Fukao et al., Phys. Lett. B 650 325-330 (2007).

[28] The RHICf Collaboration, in this proceedings. 